Linescan Camera System for $100 \%$ Moisture Measurement

J. E. Hernandez, J. Koo, C. Romero, M. Vigars, M. Newman, G. Dallum

November 7, 2006 
This document was prepared as an account of work sponsored by an agency of the United States Government. Neither the United States Government nor the University of California nor any of their employees, makes any warranty, express or implied, or assumes any legal liability or responsibility for the accuracy, completeness, or usefulness of any information, apparatus, product, or process disclosed, or represents that its use would not infringe privately owned rights. Reference herein to any specific commercial product, process, or service by trade name, trademark, manufacturer, or otherwise, does not necessarily constitute or imply its endorsement, recommendation, or favoring by the United States Government or the University of California. The views and opinions of authors expressed herein do not necessarily state or reflect those of the United States Government or the University of California, and shall not be used for advertising or product endorsement purposes.

This work was performed under the auspices of the U.S. Department of Energy by University of California, Lawrence Livermore National Laboratory under Contract W-7405-Eng-48. 


\title{
Linescan Camera System for 100\% Moisture Measurement
}

\author{
Final Report \\ Jose E. Hernandez, Jackson Koo, Carlos Romero, \\ Mark Vigars, Mike Newman, Greg Dallum \\ Lawrence Livermore National Laboratory
}

July 16,2002

\section{Executive Summary}

Lawrence Livermore National Laboratory (LLNL), in collaboration with ABB Industrial Systems, and under the sponsorship of the Department of Energy's (DOE) Office of Industrial Technologies (OIT), has developed a new method for measuring the moisture content of a paper web process on-line with $100 \%$ coverage of the sheet. The method uses InGaAs linear arrays with associated optics and electronics to continuously image the full width of the web and measure transmitted light at $1.45 \mu$ and another suitable reference wavelength between $1 \mu$ and $1.6 \mu$. The method could also be used to measure paper basis weight, in addition to moisture, by adding additional hardware and optics to measure a third wavelength at $1.57 \mu$. A patent (USP: 6355931), entitled "System and method for 100\% moisture and basis weight measurement of moving paper", was granted by the US Patent Office on March 12, 2002 for this invention. A proof-ofconcept prototype system was also developed and tested on several occasions at ABB's sensors development facility in Columbus, Ohio. Based on current experimental results, the system seems particularly suitable for detecting moisture variation on a paper web for medium and heavy weight products at the dry end as well as the press section of the machine. The prototype system was scheduled to be tested at a paper mill in the fall of 2001. The test had to be cancelled as ABB was unable to provide the required field support for the test due to restructuring and down-sizing of their R\&D organization. 


\section{Introduction}

In June 1996, several DOE National Laboratories were funded to develop sensors for the Forest Products Industry having the general objective to develop and demonstrate on-line, real-time techniques for monitoring the properties of $100 \%$ of the paper web. Three laboratories (LBNL, ANL, and PNNL) were working on fiber optic sensing systems and four laboratories (LBNL, LLNL, INEEL, and ORNL) were working on $\mathrm{CCD}^{1}$-based sensor technology. Workscopes were identified that included specific tasks at each laboratory and funding was sent through the DOE Idaho Operations Office. In February 1997, and later in October 1997, DOE-industry reviews were conducted in conjunction with the American Forest and Paper Association Sensors and Controls Task Group. After these meetings, four of the original tasks were discontinued. The remaining tasks were then individually funded from DOE-HQ through the field offices that directly oversee the laboratories. The worked described in this report is one of the continuing tasks from the original project. This report is the final report for this project and it summarizes all of the work performed since the project was started back in 1996.

\section{Problem Background}

Paper manufacturing is a very energy and waste-intensive industry. A typical paper machine makes paper that is three-hundred inches wide at speeds of up to five-thousand feet per minute. In order to produce a high quality paper product, manufacturers would like to monitor as many of the properties of a paper web as possible during manufacturing to ensure uniformity and high quality throughout the entire web. There are at least a dozen properties that are typically measured during production. Some of these include moisture, thickness, weight, formation, fiber orientation, color, and printability. Current on-line paper web measurement systems use a slow-moving scanning platform containing a suite of sensors for measuring many of these properties, including moisture (Figure 1). Moisture is a critical parameter for paper. For instance, since paper is very hydroscopic and too much moisture affects printability, manufacturers tend to take a conservative approach and over-dry their paper products, greatly increasing energy costs. Methods for making moisture measurements, based on near-infrared absorption techniques, were originally developed

1. Charged Couple Device 


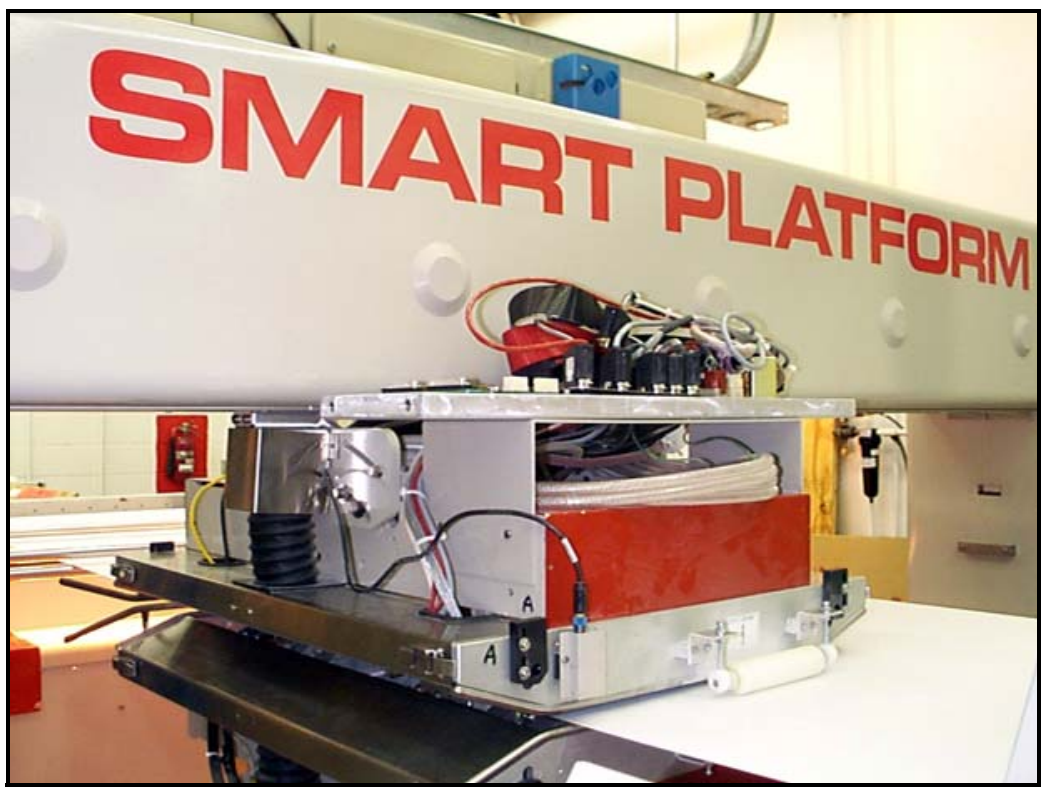

Figure 1: Commercial scanning system for measuring paper properties during production.

in the sixties, e.g., US Patent No 3,405,268. Current practices for online paper measurement of moisture and cellulose content (which is proportional to basis weight) via absorption techniques, incorporate several lead sulfide sensors for measuring wavelengths between $1.8 \mu$ and to $2.2 \mu$. These sensors are mounted on a scanning platform in close proximity to the paper web and take point measurements. Point measurements are those which only look at one "point" of approximately one inch square at any one time. This small sensing area, along with the fact that it takes about a minute or more for the scanning platform to move across the width of a typical moving paper web, can result in the production of thousands of feet of paper before a point sensor can cross the full width of the web even once. In such point measurement based systems, typically less than two percent of a total paper web area is actually measured. CCD linescan camera technology is also used on paper webs for inspection of visible defect flaws and non-uniformity detectable in the visible range (Figure 2). However, CCD sensor technology, which is based on Silicon, is not capable of detecting those wavelengths in the near-infrared range needed for accurately measuring paper moisture or cellulose content. If manufacturers had a method for conducting measurements for moisture and cellulose content over $100 \%$ of a paper web, similar to the CCD based linescan camera systems, paper-drying time could be substantially reduced, resulting 


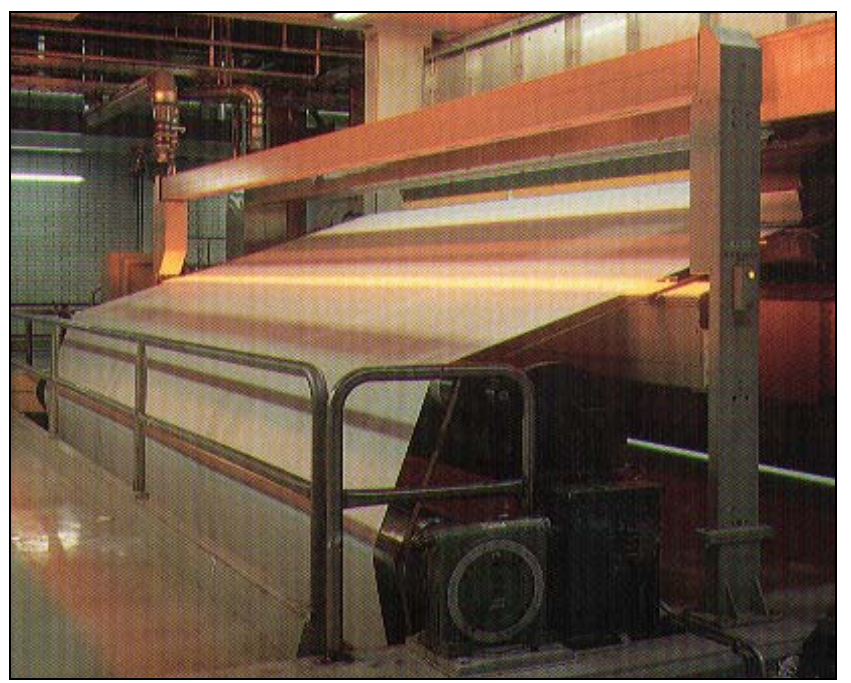

Figure 2: Commercial CCD-based system for paper web inspection of visible defects.

in significant energy savings, and improved paper quality. In response to the concerns discussed above, what is needed is a system and method for 100\% paper moisture and basis weight measurement that overcomes the limitations of current methods for measuring water and cellulose absorption.

\section{Project History and Accomplishments}

In the summer of 1996, LLNL launched a project under the sponsorship of OIT/DOE to investigate the use of linescan camera technology for $100 \%$ measurement of paper properties. During this first year, three major accomplishments were achieved: 1) development of an imaging lab at LLNL for testing a variety of camera technologies and techniques suitable for $100 \%$ measurement of paper properties (Figure 3), 2) development of a real-time data acquisition system for supporting a field test of a CCD camera system for imaging the wet end of a paper machine (Figure 4a), and 3 ) the field testing of a processing imaging system for the wet end at a paper mill (Figure 4b), which was led by Oak Ridge National Laboratory (ORNL). During this first year LLNL explored the use of color CCD linescan cameras for measuring several paper properties, including moisture, but with very little success. Based on some of this initial work, LLNL decided to focus during the following year on the development of a method and system for moisture measurement, based on the water absorption spectral line at $1.45 \mu$ 


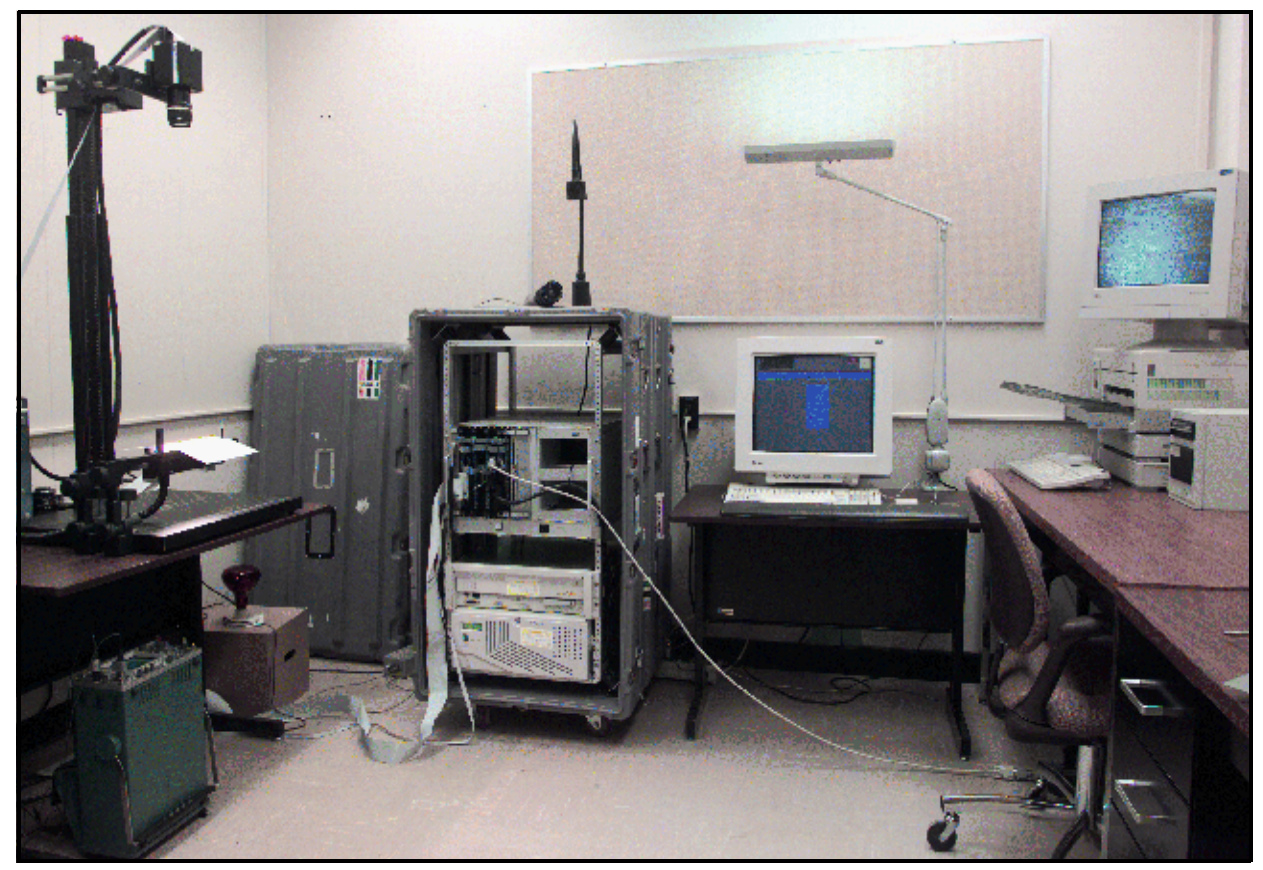

Figure 3: Paper imaging lab facility at LLNL for testing a variety of camera technologies and techniques suitable for $100 \%$ measurement of paper properties.

a)

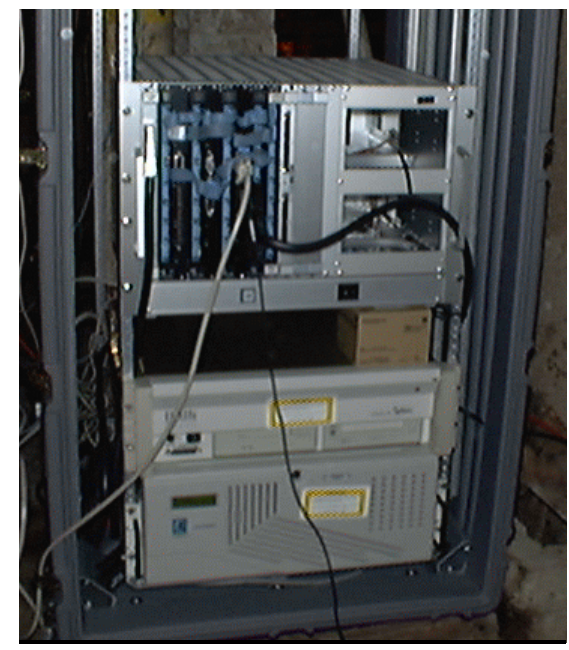

b)

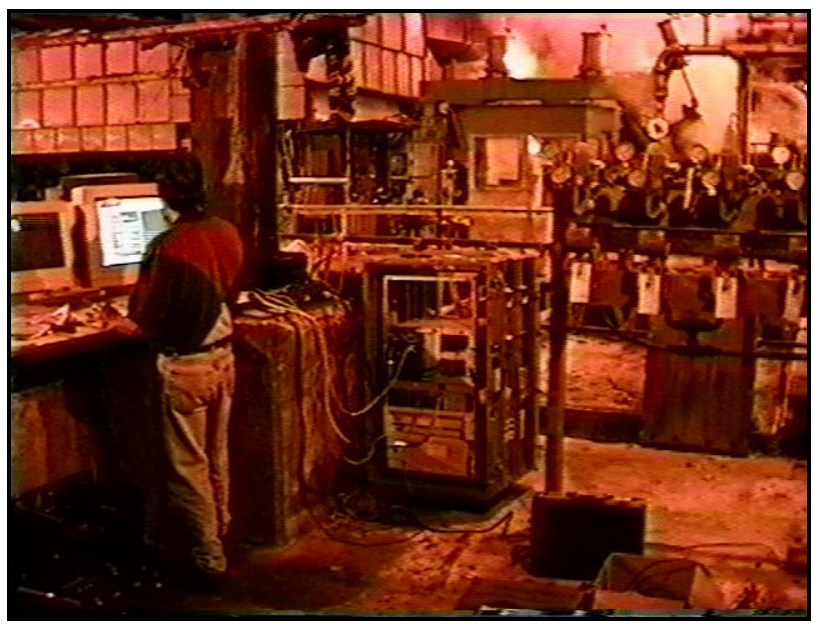

Figure 4: a) Portable real-time image processing system developed to support field testing of an imaging system for the wet end of a paper machine. The system included a host Unix workstation, a VME-based system for data acquisition and real-time processing, and a high-speed/ high-capacity disk array for real-time data recording. b) The imaging system was successfully fielded at a Union Camp paper mill in Savannah, Georgia in support of a project task led by ORNL for imaging the wet end. 
During the following year (FY97), laboratory experiments where conducted to demonstrate the feasibility of the $1.45 \mu$ wavelength for measuring water absorption. An InGaAs linescan camera was used in conjunction with a CCD linescan camera (Figure 5) for this initial work. The main motivation for using a CCD linescan camera for the reference measurement, instead of another InGaAs camera, was to reduce the cost of the system, especially in the case where a CCD-based defect inspection system was already in use at the paper machine. The main motivation for measuring water absorption at $1.45 \mu$ instead of the industry standard $1.94 \mu$, was due to the improved performance of standard InGaAs sensor relative to extended InGaAs technology, which would be required for measuring the $1.94 \mu$ wavelength. A figure of merit analysis for these two wavelengths is summarized in Table 1. In addition to the improved performance, the $1.45 \mu$ wavelength also offered the potential for making moisture measurements up-stream in the paper machine, where the $1.94 \mu$ wavelength would not work due to the large moisture content, in particular for heavy weight products. Initial laboratory results showed the feasibility of the proposed concept.

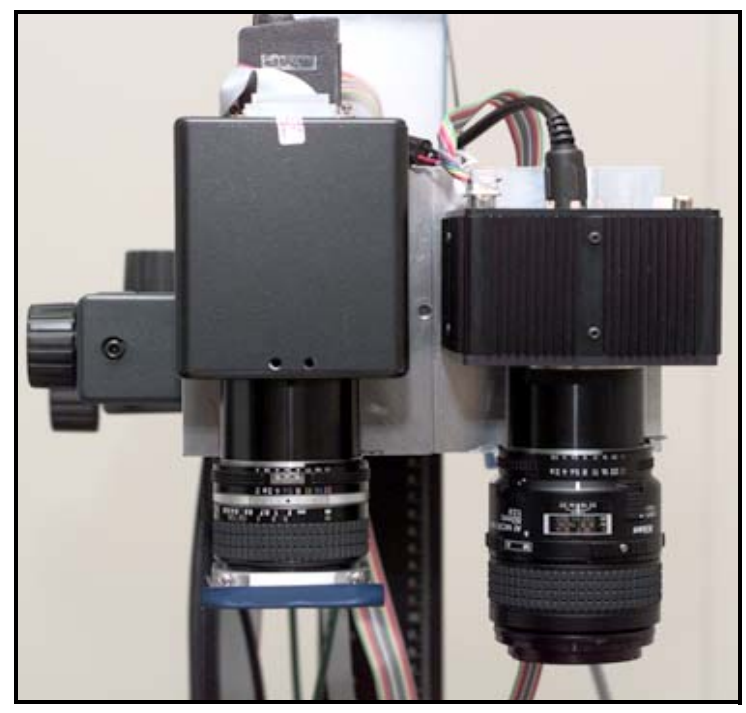

Figure 5: Initial dual-band benchtop prototype camera system for measuring moisture content consisted of a CCD linescan camera (left) for measuring light scattering in the visible range, and an InGaAs near-infrared linescan camera (right) for measuring water absorption at $1.45 \mu$. 


\begin{tabular}{|l|c|c|}
\hline \multicolumn{1}{|c|}{ Merit } & $\mathbf{1 . 4 5} \mu$ & $\mathbf{1 . 9 4} \mu$ \\
\hline \hline a) Water absorption constant & $41 \mathrm{~cm}^{-1}$ & $174 \mathrm{~cm}^{-1}$ \\
\hline b) Light intensity @ $6000^{\circ} \mathrm{K}$ & $2.5 \mathrm{Erg} / \mathrm{cm}$ & $1.0 \mathrm{Erg} / \mathrm{cm}$ \\
\hline c) Camera quantum efficiency & 0.85 & 0.75 \\
\hline d) Camera dark current & 1 & 3 \\
\hline \hline Figure of merit $\left(\mathrm{a}^{*} \mathrm{~b}^{*} \mathrm{c} / \mathrm{d}\right)$ & 87 & 43 \\
\hline
\end{tabular}

Table 1: Figure of merit analysis predicts better performance at $1.45 \mu$ relative to $1.94 \mu$ for measuring water absorption.

During the following year, FY98, other wavelengths were also evaluated, including $1.94 \mu$ which required the use of extended InGaAs sensor technology. Based on laboratory results, it was concluded that the best performance would be obtained by using the $1.45 \mu$ wavelength combined with a second InGaAs camera for the reference wavelength. This year's effort was culminated in a field demonstration of a proof-of-principle prototype system at ABB's sensors development facility in Columbus, Ohio.

This first prototype system consisted of two linescan cameras using InGaAs linear array sensors, and appropriate filters, for measuring the $1.45 \mu$ water absorption wavelength and a reference wavelength at $1.1 \mu$. A new PC-based data acquisition and processing system was developed to replace the bulky VME-based system used in the past. The camera system was mounted on ABB's testbed for evaluating sensor systems known as Matilda, which consisted of a paper winding machine and a light source (Figure 6). The testbed also had capabilities for simulating dry streak and wet streaks (Figure 7). The camera system was designed to image up to 36 " of paper (the full width of the sheet) with a resolution of 0.5 ". The system could continuously image the sheet and report a moisture profile for its full width once every second. The system was tested with three different paper products and it performed reasonably well for medium and heavy weight products. The results are summarized in Table 2. 


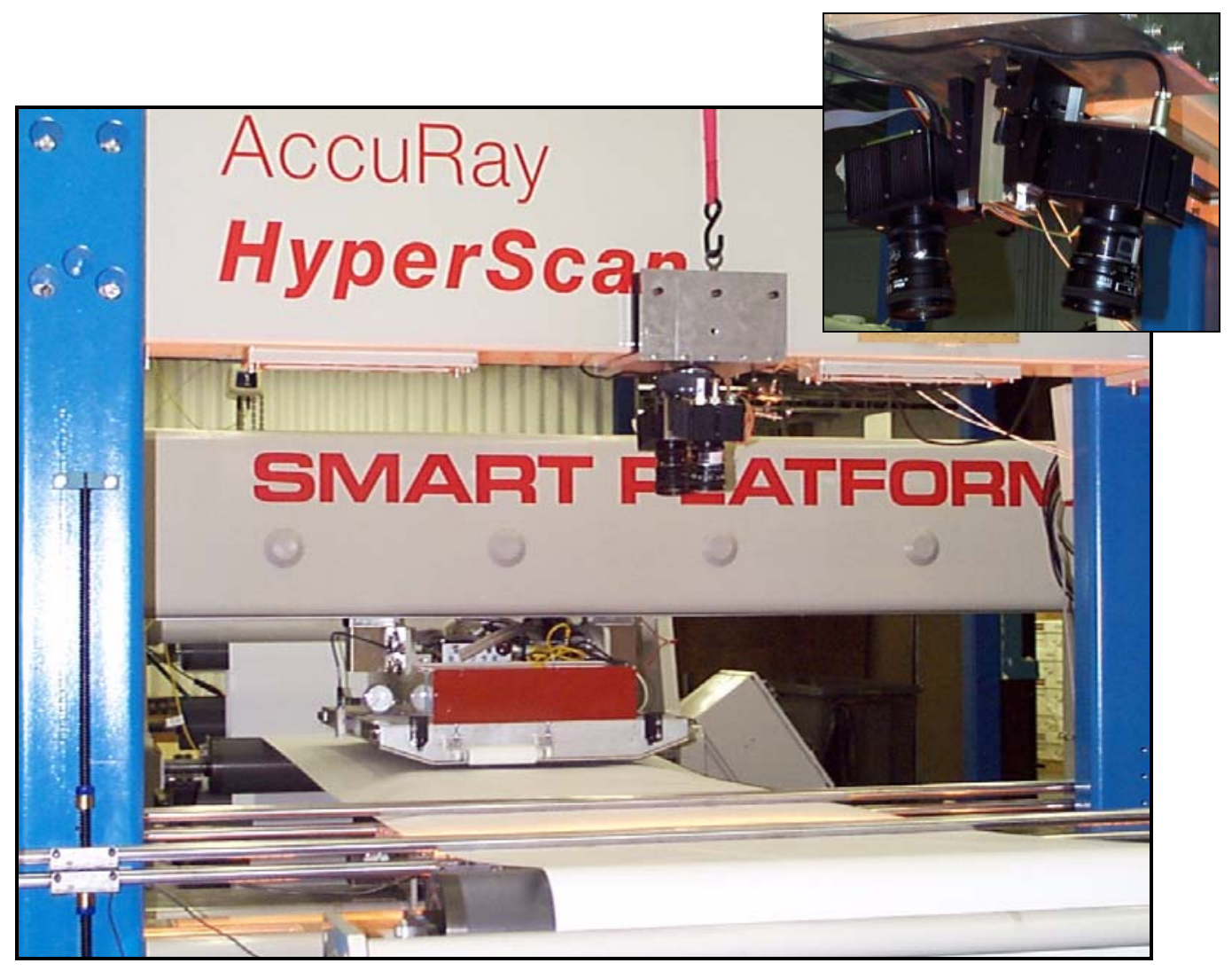

Figure 6: A proof-of-principle prototype linescan camera system for continuously measuring moisture content on moving paper was installed and tested at the Matilda testbed. The system made use of two InGaAs linescan cameras to measure transmitted light at $1.45 \mu$ and $1.1 \mu$.
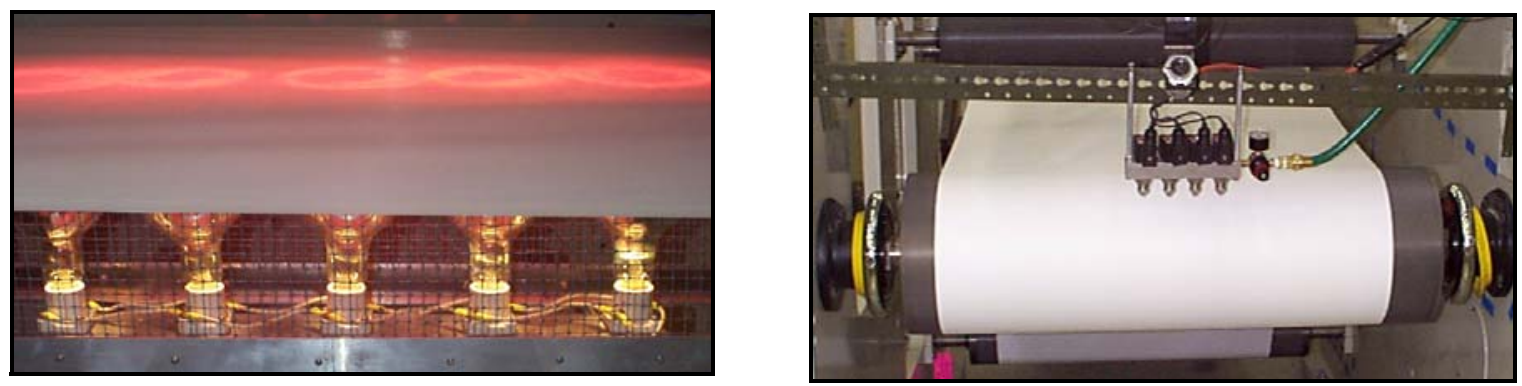

Figure 7: Heat lamps and water sprays were used to simulate dry and wet streaks during the test. 


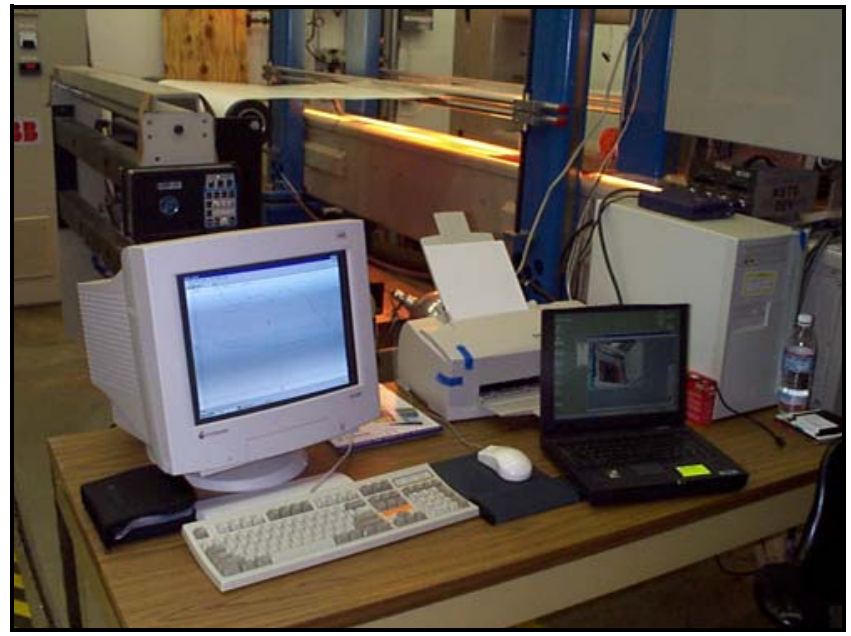

Figure 8: A new PC-based data acquisition and processing system was developed to replace the bulky VME based system used in the past.

\begin{tabular}{|l|l|l|l|}
\hline Paper Type & $\begin{array}{c}\text { Basis } \\
\text { weight }\end{array}$ & $\begin{array}{c}\text { Average } \\
\text { moisture }\end{array}$ & $\begin{array}{c}\text { System } \\
\text { sensitivity }\end{array}$ \\
\hline \hline Writing & $55 \mathrm{~g} / \mathrm{m}^{2}$ & $6.15 \%$ & $0.7 \%$ \\
\hline Wrapping & $108 \mathrm{~g} / \mathrm{m}^{2}$ & $6.3 \%$ & $0.13 \%$ \\
\hline Duplex & $188 \mathrm{~g} / \mathrm{m}^{2}$ & $7.3 \%$ & $0.05 \%$ \\
\hline
\end{tabular}

a. Estimated from experimental data

Table 2: Experimental results showed good sensitivity for medium and heavy weight paper products.

Additional improvements were proposed and implemented the following year, FY99, including the development of a single optical axis system using a dichroic mirror for reducing camera alignment errors, and repackaging of the camera system to fit inside ABB's HyperScan system (Figure 9). The improved system was tested again using the Matilda testbed during February, 2000. The new improvements resulted in an increase in system sensitivity relative to the results obtained in the previous test. Specifically, the system was able to measure dry streaks on two different paper grades with background moisture levels between $3 \%$ and $4 \%$. In contrast during the previous test, the average moisture content was 2 to 3 percent higher ( $6 \%$ to $7 \%)$, and still the system was not 
able to measure dry streaks at all. In addition, during this field test we observed very strong correlations between the moisture profiles generated by the camera system and the moisture profiles generated by the ABB scanning system based on the standard $1.94 \mu$ wavelength.

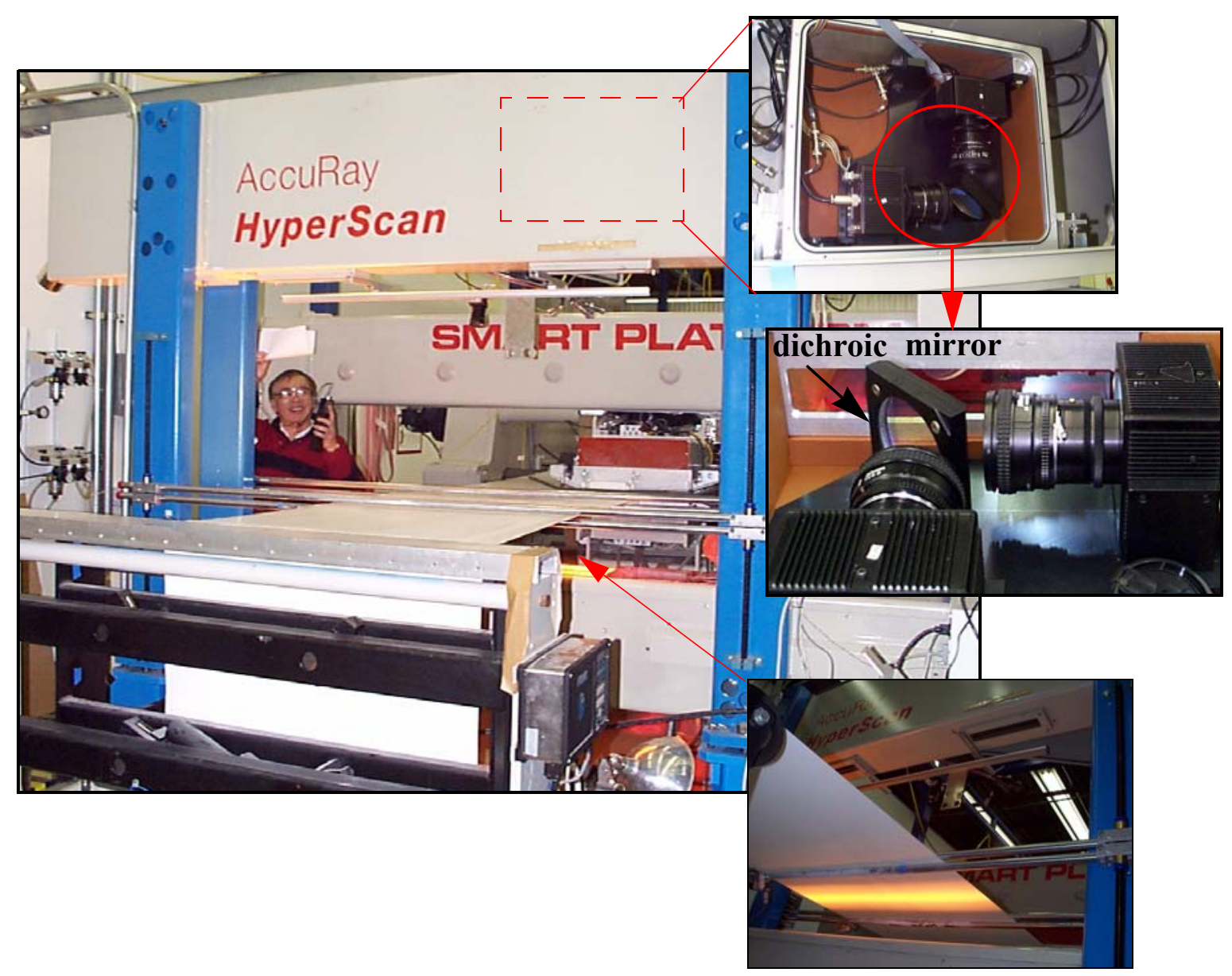

Figure 9: Improved camera system is repackaged to fit inside ABB's HyperScan system and uses a dichroic mirror for providing a single optical axis to reduce camera alignment errors.

During the second half of FY99, a new set of InGaAs linescan cameras with better sensitivity and overall performance were purchased. These new cameras were tested in the lab at ABB's facility using a set of calibrated paper samples (Figure 10). The sample set consisted of 18 different types of paper products ranging from tissue paper to $400 \mathrm{gsm}$ paper. In addition to using a $1.45 \mu$ filter for measuring water absorption, a $1.57 \mu$ filter was also used for measuring cellulose absorption, and a $1.3 \mu$ filter was used for the reference measurement, instead of the $1.1 \mu$ filter previously used. Although we only had two cameras available, we took advantage of the static nature of these tests to swap filters and perform two separate measurements per sample in order to measure 
the three wavelengths. The results, summarized in Figure 12, showed that the new system could potentially be calibrated to make absolute moisture and basis weight measurements. However, a more complete sample set and controlled experiment would be needed in the future to more accurately quantify the system's performance and ability to be calibrated to handle the large variety of paper products typically manufactured in industry. During this year, the project also received some press coverage on a local Livermore newspaper, a weekly LLNL newsletter, and a monthly DOE newsletter (Figure 11).

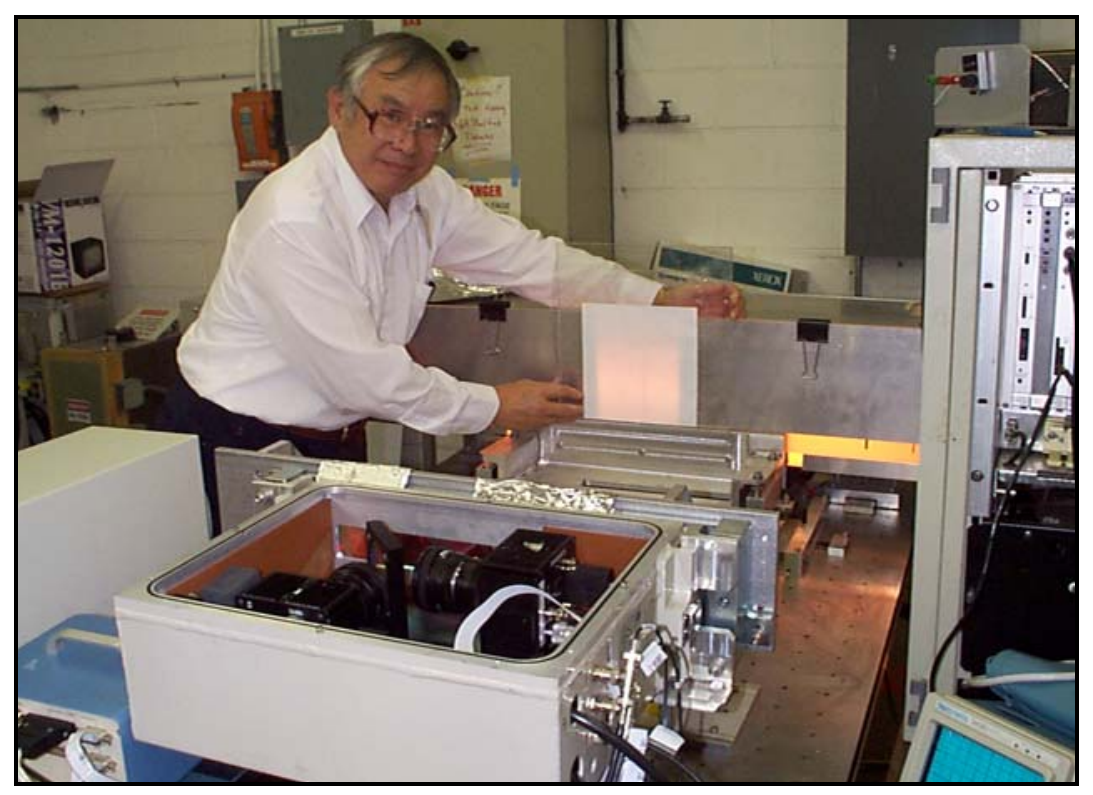

Figure 10: New InGaAs linescan cameras are tested at ABB's facility using a set of calibrated paper samples consisted of 18 different types of paper products ranging from tissue paper to 400gsm paper.
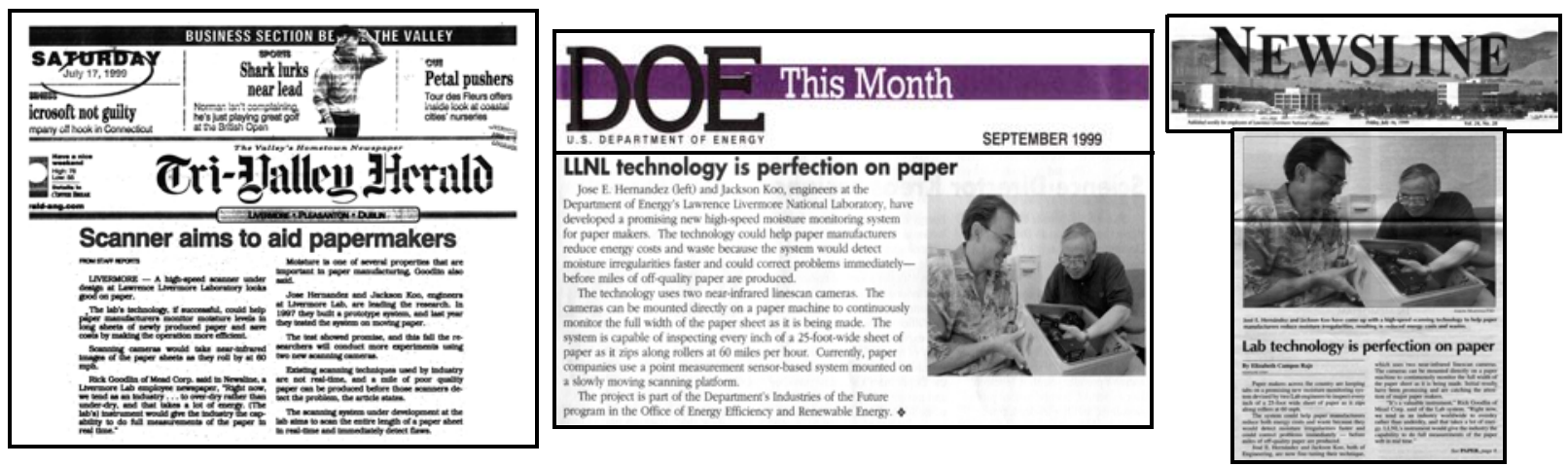

Figure 11: During 1999 the project received some press coverage in a local Livermore newspaper, a monthly DOE newsletter, and a weekly LLNL newsletter 

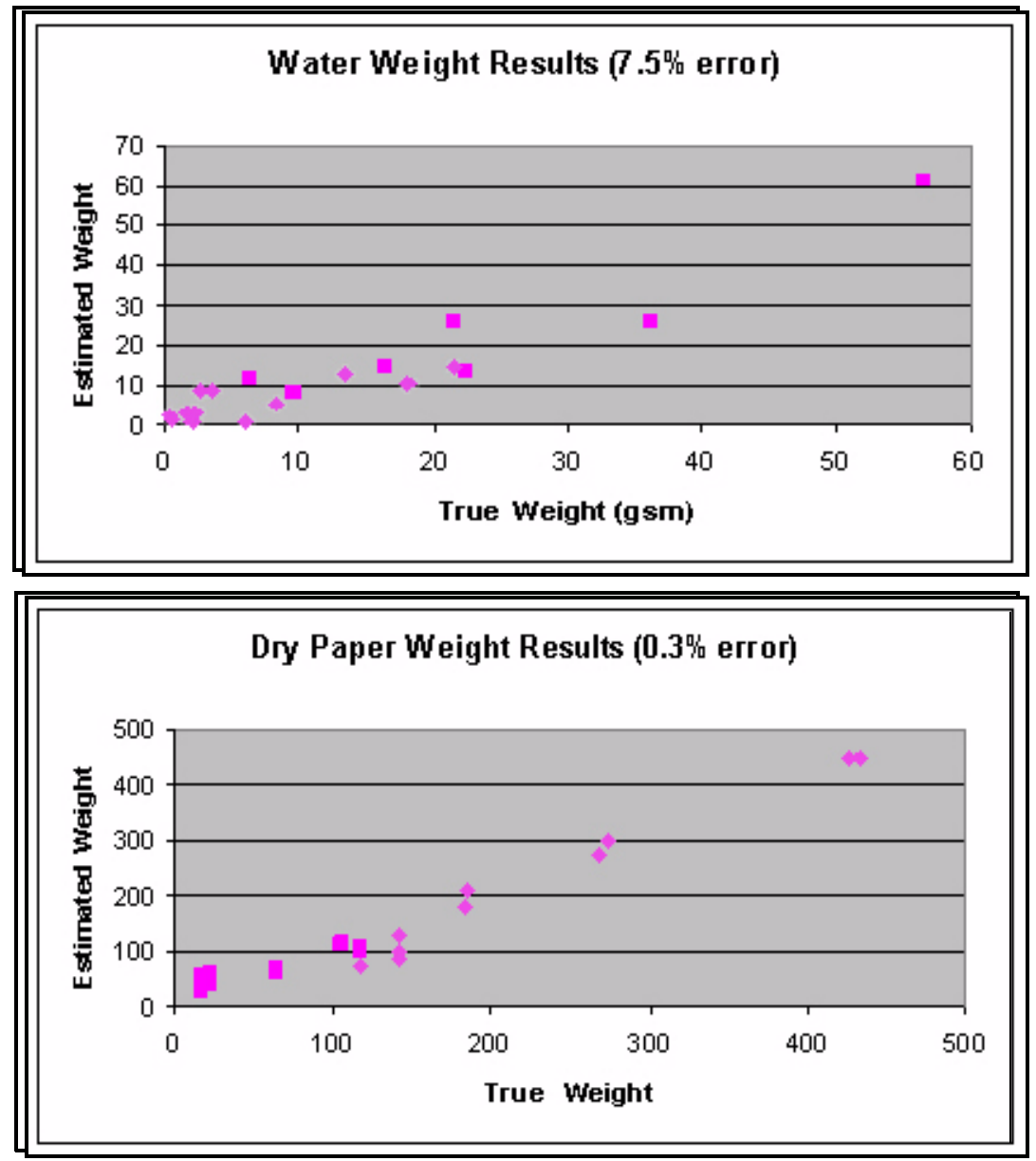

Figure 12: Laboratory results for estimating moisture content and basis weight for different paper samples using InGaAs sensor technology. Three optical narrow-band filters were used centered at $1.3 \mu, 1.45 \mu$, and $1.57 \mu$ for measuring scattering (reference), water absorption, and cellulose absorption respectively.

\section{Paper Mill Demonstration}

The last and final phase of this project was to perform a field demonstration of the prototype linescan camera system at a paper mill during FY00. An initial meeting took place in June 2000 at a Westvaco paper mill in Wickliffe, Kentucky to discuss the details and schedule of the test to take place at this mill. A series of events, primarily related to conflicts in schedules and priorities between the paper mill, ABB and LLNL caused this test to be delayed until the Fall of 2001. At the end the test had to be cancelled due to ABB not being able to provide the required field support for the test due to restructuring and down-sizing of their R\&D organization. Although the 
paper mill test was never performed, the following tasks and accomplishments were achieved during this time period:

- A new display capability was developed to visualize the moisture profiles as a two-dimensional color map representing the sheet, and using different colors to show problem areas (Figure 13).

- The field of view for the camera's single optical axis system was increased from 18 inches to 1 meter to support the field test.

- The camera interface electronics was modified and repackaged to fit inside the HyperScanner case provided by ABB (Figure 14).

- The power supplies for the cameras and interface electronics were enclosed in a ruggedized and watertight case (Figure 14).

- The interface panel on the camera system enclosure was modified to ensure it was watertight and support air cooling via an external air hose.

- An entry package was completed and submitted jointly with ABB for nominating the new linescan camera system for an R\&D100 Award.

- A patent was granted by the US Patent Office for this work on March 2002.

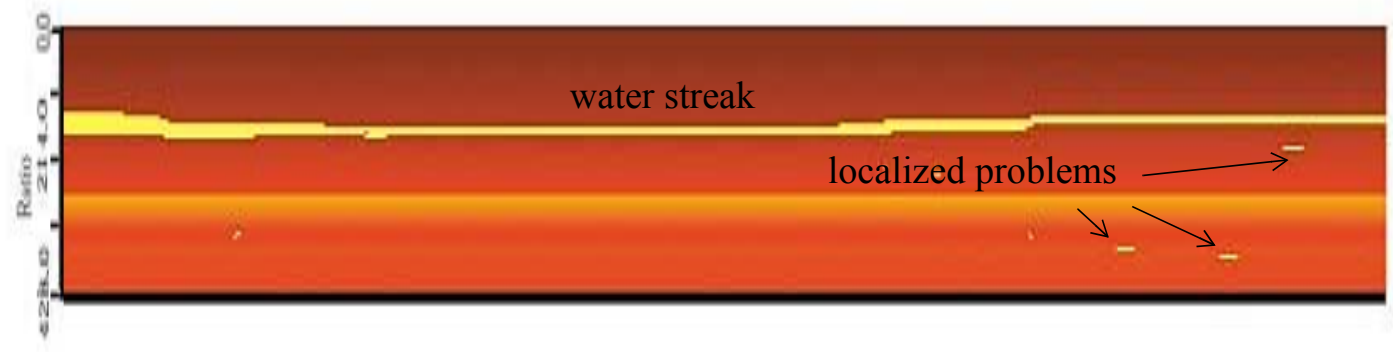

Figure 13: New 2-D display capability for visualizing the moisture profiles of the sheet. The above photo shows an on-going wet streak (yellow horizontal line) as well as some other localized problems. 

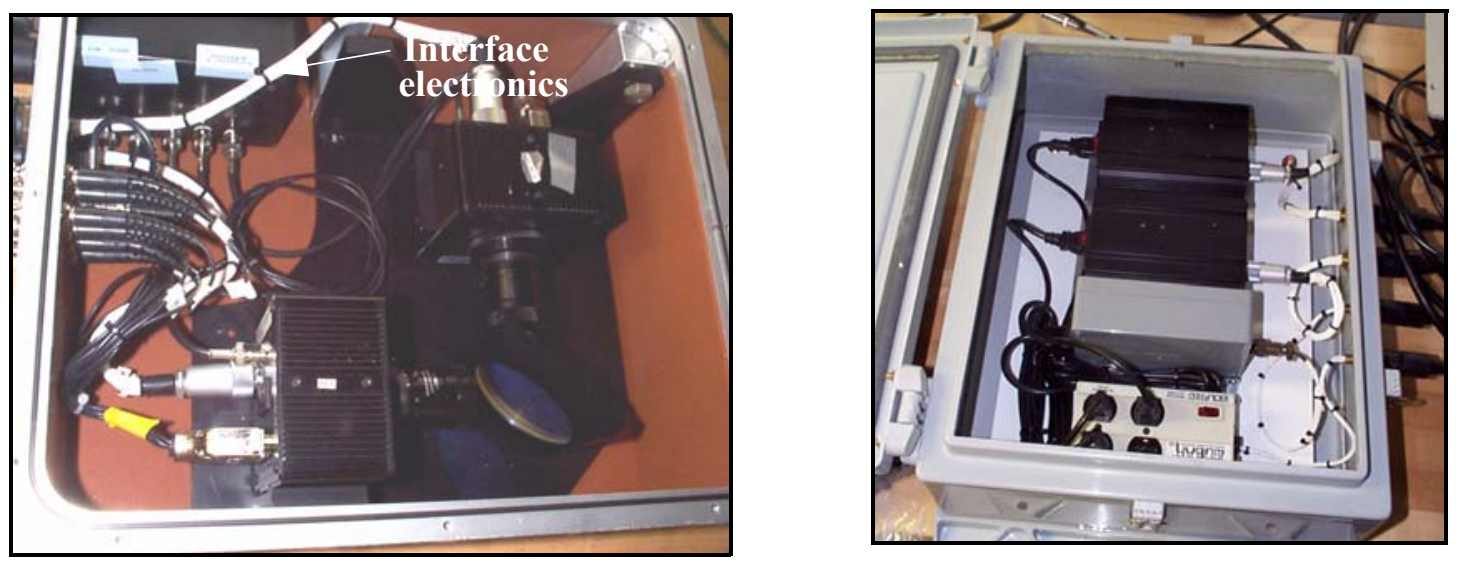

Figure 14: Camera interface electronics was modified and repackaged to fit inside the HyperScanner case, power supplies are repackaged in a ruggedized and watertight case.

\section{Conclusions}

A new method was developed for measuring the moisture content of a paper web process on-line with $100 \%$ coverage of the sheet. The new method uses InGaAs linear arrays with associated optics and electronics to continuously image the full width of the web and measure transmitted light at $1.45 \mu$ and another suitable reference wavelength between $1 \mu$ and $1.6 \mu$. The method can also be used to measure paper basis weight in addition to moisture by adding additional hardware to measure a third wavelength at $1.57 \mu$. A patent (USP: 6355931) entitled "System and method for $100 \%$ moisture and basis weight measurement of moving paper", was granted by the US Patent Office on March 12, 2002 for this invention. A proof-of-concept prototype system was also developed and tested on several occasions at ABB's sensors development facility in Columbus, Ohio. Based on laboratory experimental results, the system seems particularly suitable for detecting moisture variations on a paper web for medium and heavy weight products at the dry end as well as the press section of the machine. The new patent has been added to LLNL's portfolio of technologies with commercial potential and it is available for licensing.

\section{Acknowledgements}

The authors acknowledge and thank ABB Industrial Systems for letting us use their facilities and providing support personnel for helping with the testing of the prototype system. In particular we thank Dr. Gary Burk for all the valuable help and guidance he provided to this project. 